Modeling, Identification and Control, Vol. 30, No. 3, 2009, pp. 181-190, ISSN 1890-1328

\title{
Trends in Research and Publication: Science 2.0 and Open Access
}

\author{
Morten Breivik ${ }^{1}$ Geir Hovland ${ }^{2}$ Pål Johan From ${ }^{3}$
}

\author{
${ }^{1}$ Centre for Ships and Ocean Structures, Norwegian University of Science and Technology, NO-7491 Trondheim, \\ Norway. E-mail: morten.breivik@ieee.org \\ ${ }^{2}$ Department of Engineering, Mechatronics Group, University of Agder, NO-4898 Grimstad, Norway. E-mail: \\ geir.hovland@uia.no \\ ${ }^{3}$ Department of Engineering Cybernetics, Norwegian University of Science and Technology, NO-7491 Trondheim, \\ Norway. E-mail: from@itk.ntnu.no
}

\begin{abstract}
This paper considers current trends in academic research and publication, in particular as seen from the control community. The introduction of Web 2.0 applications for scientists and engineers is currently changing the way research is being conducted. In the near future, participants in the research community will be able to share ideas, data and results like never before. They will also be able to manage the rapidly increasing amount of scientific information much more effectively than today through collaborative efforts enabled by the new Internet tools. However, an important premise for such a development is the availability of research material. Many research results are currently shielded behind expensive subscription schemes that impede the sharing of information. At the same time, an increasing amount of research is being published through open access channels with unrestricted availability. Interestingly, recent studies show that such policies contribute to an increased number of citations compared to the pay-based alternatives. In sum, the parallel development of new tools for research collaboration and an increased access to research material may fundamentally transform the way research is going to be conducted in the future.
\end{abstract}

Keywords: Web 2.0, Science 2.0, Open Access, Journal Ranking, Print On Demand, Publication Methods

\section{Introduction}

Many important activities related to science and research are currently located in the offline world of yesterday and have not kept up with an increasingly online reality. The possibilities offered by the Internet and its World Wide Web have not been fully taken advantage of by the scientific communities around the world, including those involved with the fields of modeling, identification and control. Although on a digital format, most new research results are still reported through traditional outlets as if paper was the only publication medium. Despite having access to e-mail and instant messaging, most researchers are still collab- orating within relatively small and confined research groups. Situated at the beginning of the 21st century, it seems strange that key scientific activities such as those related to publication and collaboration haven't changed much since the middle of the 20th century.

At the same time, a few large publishing conglomerates have spent the last decades buying up small but prestigious journals in order to create monopoly-like situations regarding research material. This situation has enabled them to significantly increase the subscription fees for providing access to such material to universities and research institutions. Through a remarkable publication cycle, work primarily funded by the public thus ends up not being publicly available. 
Enter Science 2.0 and Open Access. Promising to address the above-mentioned issues by unleashing the full potential of the Internet, these concepts are chosen as the topics of this paper, whose goal is to introduce the control community to exciting new developments which can fundamentally change our work day. Keywords are access, collaboration and content.

\section{Science 2.0}

Wikipedia defines the term Web 2.0 as “... commonly associated with web applications which facilitate interactive information sharing, interoperability, usercentered design and collaboration on the World Wide Web." (Wikipedia, 2009a). Relevant examples of such applications include blogs, social-networking sites, video-sharing sites and wikis. These websites allow users to change their content and interact with other users, as opposed to just being passive receivers of provided information. In fact, the user-generated encyclopedia Wikipedia itself represents one of the most used Web 2.0 applications around.

\subsection{Web 2.0}

The most popular social-networking site in the world today is Facebook, which currently has over 300 million active users. These users can create their own, personal profiles where they can publish information about themselves. However, the most popular features relate to how they can interact with each other by exchanging messages, sharing pictures and videos, playing games, etc.

LinkedIn represents the business-equivalent of Facebook and is used for professional networking. It provides its current user base of over 50 million people the possibility of creating personal profiles and sharing work-related information such as job and business opportunities. Companies and professional organizations also have a presence on LinkedIn.

Among the video-sharing sites, YouTube tops the list. It lets users upload, view and share videos, and has revolutionized the way in which such content has become available on the web. In 2008, YouTube was awarded a Peabody Award as "... an ever-expanding archive-cum-bulletin board that both embodies and promotes democracy." (Wikipedia, 2009b).

Other popular and interactive websites include the Internet Movie Database (IMDb), which provides online information about movies, televisions shows and actors. Registered users are able to rate movies and write reviews, which other users find useful when evaluating whether to watch a specific movie or not.
What all these websites have in common is the framework they provide for ordinary people to easily generate and share content with each other within specific areas of interest. This feature is also the key to their enormous success. As pointed out by Harvard Law School professor and Creative Commons cofounder Lawrence Lessig, this development enables the return of a read-write culture, as opposed to the readonly culture that dominated the 20th century (Lessig, 2009). People no longer only consume but also become producers of creative content.

How does this web-based development relate to the world of academic research and publication, where scientists have always been both consumers and producers? It turns out that it represents a unique opportunity which is just beginning to take shape.

\subsection{Web 2.0 for Scientists}

In 1945, having coordinated the activities of 6000 leading American scientists during the Second World War, the great science administrator Vannevar Bush wrote (Bush, July 1945): "There is a growing mountain of research. But there is increased evidence that we are being bogged down today as specialization extends. The investigator is staggered by the findings and conclusions of thousands of other workers - conclusions which he cannot find time to grasp, much less to remember, as they appear." Continuing: "Professionally our methods of transmitting and reviewing the results of research are generations old and by now are totally inadequate for their purpose. If the aggregate time spent in writing scholarly works and in reading them could be evaluated, the ratio between these amounts of time might well be startling." Then concluding that: "The difficulty seems to be ... that publication has been extended far beyond our present ability to make real use of the record."

To help solve such problems, Bush suggested the development of a device he called a "memex", coined as a combination of the words "memory" and "index", which he defined as "... a device in which an individual stores all his books, records, and communications, and which is mechanized so that it may be consulted with exceeding speed and flexibility."

Today, scientists have much easier access to research material than in 1945, in particular due to the Internet and its World Wide Web, which provides memex-like services to all. Conferences and journals make their content available online for convenient and easy download for subscribers, and search engines such as Google make it easier than ever to find new material.

However, the amount of material is also growing as never before. With an emphasis on quantity over quality, current financing schemes encourage scientists to 
publish more often and with fewer increments in results between successive publications.

With such opposite trends working against each other, the challenge for any scientist or researcher becomes the same as in Bush's days: To locate relevant literature as fast as possible.

In solving this problem, inspiration can be sought from the dominating Web 2.0 applications of today. Imagine a mix of Facebook, LinkedIn, YouTube and IMDb, only specifically tailored for scientists. A framework for user-generated content, supplied by the scientists themselves. A way for scientists to conveniently share and organize information about their work. A tool for them to collaborate in classifying, reviewing and managing the large and rapidly increasing body of research material in their respective fields of work.

Launched in 2005 by graduate students collaborating on synthetic biology, the MIT-based OpenWetWare project shows how Web 2.0 infrastructure can be used advantageously for research purposes (Waldrop, 2008). A collaborative wiki, OpenWetWare has rapidly grown into a social network serving a broad range of biologists and biological engineers. Laboratories on five continents currently use the website, it encompasses dozens of courses and interest groups, and hundreds of discussions about lab protocols and techniques are ongoing. According to lab manager Maureen Hoatlin: "I came to love the interaction, the fact that people in other labs could comment on what we do and vice versa. When I see how fast that is, and its power to move science forward-there is nothing like it."

Adressing concerns about possible vandalism, OpenWetWare allows users to make changes only after they have registered and established their affiliation with a legitimate research institution. Regarding the danger of getting scooped by someone stealing ideas from a wiki, chemist Jean-Claude Bradley believes that: "The Web provides better protection than the traditional journal system. Every change on a wiki gets a timestamp, so if someone actually did try to scoop you, it would be very easy to prove your priority-and to embarrass them. I think that's really what is going to drive open science: the fear factor. If you wait for the journals, your work won't appear for another six to nine months. But with open science, your claim to priority is out there right away."

More recent initiatives include the scientific networking site ResearchGATE, which was launched in 2008 and whose underlying motivation is stated as: "The vision of Science 2.0 is promising: Communication between scientists will accelerate the distribution of new knowledge. ... Science is collaboration, so scientific social networks will facilitate and improve the way scientists collaborate." Another website in the same vein is Mendeley, which started out as a tool for managing and sharing research papers, but now also has evolved into providing online collaboration and socialnetworking features for researchers. Additional facilitators for web-based communication and collaboration include Google Wave, which is designed as a mix between e-mail and instant messaging. According to Google, a wave "... can be both a conversation and a document where people can discuss and work together using richly formatted text, photos, videos, maps, and more."

Combined, these tools can help scientists both manage their research literature better as well as connect and interact efficiently with colleagues all around the world. The sharing of ideas, data and results represents one of the key cornerstones of science, and the rapid development of Web 2.0 applications for scientific purposes enables more efficient sharing than ever before. Ultimately, the development of Science 2.0 applications recognizes the fact that individual researchers are collectively better equipped to organize information and connect the important dots than a few selected professionals who work full time with such endeavors but who have obvious restrictions on their level of overview, personal preferences and work capacity.

Science 2.0, also frequently referred to as open science or open research, is nevertheless still only in its infancy. However, according to online community manager Bora Zivkovic at the open access resource PLoS ONE, experiments in Science 2.0 are currently proliferating so fast that it's impossible to keep track of them (Waldrop, 2008): "It's a Darwinian process. About 99 percent of these ideas are going to die. But some will emerge and spread." Cancer researcher Bill Hooker adds: "I wouldn't like to predict where all this is going to go. But I'd be happy to bet that we're going to like it when we get there."

\section{Open Access}

"Writing researchers get paid by the public, their editors get paid by the public. Afterwards, the publishers are left with the copyright to the research articles." This citation is taken from a news article in the Norwegian newspaper Aftenposten focusing on the current scientific publication regime (Bjørkeng, 2009). According to the article, it is not unusual that the largest scientific publishing houses achieve a $30 \%$ profit of their turnover. In comparison, the rest of the publishing business must typically settle with $3 \%$.

During recent years, the largest academic publishers have significantly turned up the price of their services, forcing university libraries to spend up to $70-80 \%$ of their budgets on electronic resources. According to 
(Gripsrud, 2006), the University of Bergen now spends as much as $99 \%$ of its purchasing budget in medicine and natural science on electronic journal subscriptions. Librarians Kari Garnes and Ole Evensen comment on the development: "We are concerned ... The journals are eating up our economy while also putting severe limitations on our rights." In the period 1990-2005, the price of the major journals increased by $200 \%$ while the price index only rose by $57 \%$ (Gripsrud, 2006).

According to librarian Leif Longva at the University of Troms (Bjørkeng, 2009): "The problem is that journal publishing in academia is still stuck in a model that goes back to the time of paper-based journals. Then it was quite reasonable with subscriptions. However, when publishing on the web, there is no reason why anybody who wants access to scientific articles shouldn't get it. It's primarily publicly funded research we are talking about here, and then it is optimal, seen from the viewpoint of society, that the cost of reading is zero."

What Longva is calling for is referred to as open access (OA) publishing. In general, OA means free access to any kind of electronic material available on the Internet. In a scientific context, OA publications typically refer to freely available scholarly work as represented by scientific articles and books. Such publications can thus be downloaded by anyone, anywhere, anytime, which matches perfectly with the collaborative vision of Science 2.0.

\subsection{Open Access and Science 2.0}

A necessary condition for the arrival and impact of Science 2.0 is that scientists are allowed to publish and exchange their research material freely. As already mentioned, most of their scientific output is currently published through channels with restricted access, requiring increasingly expensive subscriptions to gain admission. This regime means that researchers working at institutions which cannot afford the subscription fees are effectively left out in the cold. In particular, this situation affects researchers in developing countries.

However, although OA is necessary for Science 2.0, Science 2.0 will also support OA. When scientists catch on and increasingly start using the new web-based applications as part of their daily routine, they will see the need for and demand an increase in freely available material. Science 2.0 and OA will thus help push each other forward to the benefit of both.

\subsection{Open Access Publications}

At the moment, open access publications can be divided into two main categories:

1. Electronic archives established by individual uni- versities or research institutions which offer open access to the publications of their employees.

2. Peer-reviewed electronic journals which provide freely available articles for download.

Regarding institutional archives, two main factors currently limit their use. First, the institutions need exemption from the copyright of the publishers in order to provide free access to the articles. However, today over $90 \%$ of journals permit such archiving (Hugubakken, 2006). Second, encouragement to deposit articles in institutional archives has so far not been effective. International experience shows that requirements will have a much larger impact (Norges Forskningsråd, 2009).

Regarding newly established OA journals, a limiting factor is their acceptance as valid publication channels. The Norwegian DBH database (Database for statistikk om høgre utdanning) lists all accepted academic publication channels in Norway. As of June 2009, only about 150 of a total of 23000 publication channels were registered as open access. On the other hand, the Directory of Open Access Journals (DOAJ) currently lists more than 4300 OA journals worldwide. While it is expected that the number of $\mathrm{OA}$ journals will increase sharply in the future, the increase of such journals in the DBH database is predicted to be smaller. It takes time for a new journal to build its reputation, especially since most researchers are required by their institutions to publish in journals that are already accepted as valid publication channels. The growth of OA journals in the DBH database is thus more likely to come from established journals converting to open access.

\subsection{Funding of Open Access}

In the coming years, national funding agencies are expected to play a more important role regarding open access policies by demanding that publicly funded research should be made freely available. As an example, Det Frie Forskningsråd | Kultur og Kommunikation (FKK) in Denmark already requires journals receiving funding to become OA within one year. Also, the Swedish research council Vetenskapsrådet recently announced that all researchers who receive funding must guarantee that their publications are available as OA within maximum six months of publication. Similarly, on January 29th 2009 the Norwegian Research Council (Norges forskningsråd, NFR) announced new OA guidelines for scientific publications, where all funded researchers are required to make their results accessible either through instutional archives or through OA journals (Norges Forskningsråd, 2009). Institutional libraries have already supported OA for many years since its growth will help reduce their subscription fees. 
On September 14th 2009, the 5 US universities Cornell, Dartmouth, Harvard, Massachusetts Institute of Technology (MIT) and the University of California, Berkeley (UCB) announced joint support for OA publications (Richards, 2009). According to Thomas Leonard, librarian at UCB: "Publishers and researchers know that it has never been easier to share the best work they produce with the world. But they also know that their traditional business model is creating new walls around discoveries. Universities can really help take down these walls and the OA compact is a highly significant tool for the job."

The recent economic downturn also underlines the importance of OA publications. With budget cuts straining library resources, subscription and licensing fees for journals have come under increasing scrutiny. Alternative means for providing access to vital intellectual content are thus explored. According to Harvard computer science professor Stuart Shieber (Richards, 2009): "Universities and funding agencies ought to provide equitable support for OA publishing by subsidizing the processing fees that faculty incur when contributing to OA publications. Right now, these fees are relatively rare. But if the research community supports OA publishing and it gains in importance as we believe that it will, those fees could aggregate substantially over time. The compact ensures that support is available to eliminate these processing fees as a disincentive to $O A$ publishing."

However, the future funding mechanisms for OA publications remain an open issue. With a full transition to OA, publication costs must be shifted from the subscribers to either the funding agencies, research institutions, authors or to other sponsors. It will be a vital challenge in the future to ensure that such costs do not increase to a level where they prevent certain scientists and researchers from publishing their work in high-rank journals in the same way that large subscription fees prevent some of them access to the work of their colleagues today.

\subsection{Journal Ranking and Citations}

An increasing trend in previous years is to quantify and rank the research impact of both journals and individual researchers. Two common measures are the ISI Impact Factor and the h-index.

The ISI Impact Factor for a given year is a measure of the average number of citations per article for a journal during the previous two years, and the idea dates back to Garfield (1955). The h-index is more recent and can be defined as follows (Hirsch, 2005): A journal has index $h$ if $h$ of all $N_{\mathrm{p}}$ papers have at least $h$ citations each, and the other $\left(N_{\mathrm{p}}-h\right)$ papers have at most $h$ citations each. While the impact factor is most commonly used for journals, the h-index is used for both journals and individual researchers.

Table 1 shows some selected journals in the field of automation and control ranked by the h-index. For example, Automatica has a h-index of 124, which means that 124 articles have been cited 124 times or more. The h-index is a more stable measure of journal impact than the ISI Impact Factor, which only considers citations during the last two years. Also, while the impact factor can change significantly between two consecutive years, the h-index can never decrease. Note that MIC's impact factor has not been calculated in 2007 and 2008, but will reappear in 2009 .

Table 2 shows the most cited papers during the last decade from the top 10 journals listed in Table 1 . Not surprisingly, the highest ranked journals also have the most cited papers. The research topics dominating the citations in the area of automation and control during the last decade include control of constrained systems, multi-agent systems and time-delay systems.

Since 2005, a significant amount of the research funding at Norwegian universities has been directly linked to how much the academic staff publish in certain accredited journals. As mentioned earlier, the DBH database lists all the accepted publication channels in Norway. The channels are ranked in an incentive hierarchy based on data from the ISI Web of Knowledge, using measures such as the ISI Impact Factor. Naturally, individual scientists and researchers aim at advancing their own careers by publishing in the highest ranked journals. They cannot afford to publish in newly established OA journals with low ranking on purely idealistic grounds. The current incentive system thus favors the established journal hierarchy and prevents the growth of OA publications, thereby contradicting the new OA requirements imposed by the research council NFR.

\subsection{Open Access and Citations}

The effect of open access on the number of citations has received a lot of attention after the OA journals appeared on the web in the 1990s. Most reports tend to support the view of an OA citation advantage, which claims that OA publications cause more citations than comparable non-OA publications.

Early work supporting this citation advantage is presented in (Lawrence, 2001), where it is found that conference papers which are freely available for download have greater impact than those that are not. A significant $150 \%$ citation advantage is claimed. A possible explanation for this finding might be that freely distributed papers are more visible. As access becomes more convenient, papers are more frequently downloaded and their usage increases. This effect helps the 
Modeling, Identification and Control

\begin{tabular}{|c|c|c|c|c|c|c|c|}
\hline$\#$ & Journal & $\mathbf{h}$ & IF08 & TA & TC & TC/TA & DBH \\
\hline$\overline{1}$ & IEEE Transactions on Automatic Control* & 134 & 3.293 & 6208 & 121613 & 19.59 & 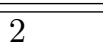 \\
\hline 2 & Automatica & 124 & 3.178 & 5960 & 111492 & 18.71 & 2 \\
\hline 3 & IEEE Trans. on Systems, Man and Cybernetics & 95 & 2.361 & 3507 & 72774 & 20.75 & 2 \\
\hline 4 & SIAM Journal on Applied Mathematics & 91 & 1.110 & 4556 & 64636 & 14.19 & 2 \\
\hline 5 & International Journal of Control & 89 & 1.130 & 7768 & 77733 & 10.01 & 1 \\
\hline 6 & Chemometrics and Intelligent Laboratory Systems & 73 & 1.940 & 2351 & 31670 & 13.47 & 1 \\
\hline 7 & IEEE Transactions on Industrial Electronics & 67 & 5.468 & 4008 & 43089 & 10.75 & 1 \\
\hline 8 & Systems \& Control Letters & 66 & 2.073 & 2311 & 28632 & 12.39 & 2 \\
\hline 9 & SIAM Journal on Control and Optimization & 61 & 1.517 & 1995 & 24637 & 12.35 & 2 \\
\hline 10 & Journal of Optimization Theory and Applications & 60 & 0.860 & 4393 & 33669 & 7.66 & 1 \\
\hline 11 & IEEE Transactions on Control Systems Technology & 50 & 2.130 & 1406 & 16321 & 11.61 & 1 \\
\hline 12 & IEEE Control Systems Magazine & 48 & 2.827 & 1105 & 11202 & 10.14 & 1 \\
\hline 13 & ASME Journal of Dyn. Sys. Measurement and Control & 48 & 0.753 & 2797 & 19502 & 6.97 & 1 \\
\hline 14 & IEEE-ASME Transactions on Mechatronics & 40 & 1.614 & 846 & 8934 & 10.56 & 1 \\
\hline 15 & IEE Proceedings - Control Theory and Applications & 39 & 2.106 & 1076 & 9849 & 9.15 & 1 \\
\hline 16 & International Journal of Robust and Nonlinear Control & 39 & 1.560 & 1130 & 9249 & 8.18 & 1 \\
\hline 17 & Robotics and Autonomous Systems & 38 & 1.214 & 1231 & 10367 & 8.42 & 1 \\
\hline 18 & Journal of the Franklin Inst. - Eng. and Appl. Math. & 38 & 0.616 & 3639 & 13568 & 3.73 & 1 \\
\hline 19 & Journal of Machine Learning Research & 37 & 3.116 & 626 & 7538 & 12.04 & 2 \\
\hline 20 & Control Engineering Practice & 37 & 1.871 & 2218 & 14166 & 6.39 & 1 \\
\hline 21 & International Journal of Systems Science & 37 & 0.634 & 4815 & 17773 & 3.69 & 1 \\
\hline 22 & Journal of Process Control & 36 & 1.606 & 990 & 9126 & 9.22 & 2 \\
\hline 23 & Mathematics of Control Signals and Systems & 28 & 1.500 & 321 & 3757 & 11.70 & 1 \\
\hline 24 & Engineering Applications of Artificial Intelligence & 27 & 1.397 & 1310 & 5956 & 4.55 & 1 \\
\hline 25 & International Journal of Advanced Manufacturing & 27 & 0.743 & 4287 & 13302 & 3.10 & 1 \\
\hline 26 & IEEE Robotics \& Automation Magazine & 26 & 3.000 & 594 & 3177 & 5.35 & 1 \\
\hline 27 & Mechatronics & 26 & 1.434 & 1115 & 5818 & 5.22 & 1 \\
\hline 28 & IEEE Transactions on Robotics & 24 & 2.656 & 630 & 3156 & 5.01 & 1 \\
\hline 29 & Int. Journal of Adaptive Control and Signal Proc. & 24 & 1.403 & 818 & 4078 & 4.99 & 1 \\
\hline 30 & Autonomous Agents and Multi-Agent Systems & 22 & 2.125 & 234 & 1934 & 8.26 & 1 \\
\hline 31 & Discrete Event Dyn. Systems - Theory and Appl. & 21 & 0.974 & 263 & 1755 & 6.67 & 1 \\
\hline 32 & Optimal Control Applications \& Methods & 20 & 0.833 & 747 & 2875 & 3.85 & 1 \\
\hline 33 & European Journal of Control & 19 & 1.013 & 663 & 1608 & 2.43 & 1 \\
\hline 34 & ESAIM: Control Optimisation and Calculus of Var. & 18 & 0.787 & 385 & 1775 & 4.61 & 1 \\
\hline 35 & Journal of Vibration and Control & 18 & 0.656 & 861 & 2596 & 3.02 & 1 \\
\hline 36 & SIAM Journal on Applied Dynamical Systems & 17 & 1.211 & 253 & 1219 & 4.82 & 1 \\
\hline 37 & Control and Cybernetics & 16 & 0.689 & 589 & 1288 & 2.19 & 1 \\
\hline 38 & Proc. of the Inst. of Mechanical Engineers - Part I & 15 & 0.322 & 740 & 1647 & 2.23 & 1 \\
\hline 39 & Trans. of the Institute of Measurement and Control & 14 & 0.500 & 441 & 1027 & 2.33 & 1 \\
\hline 40 & Modeling, Identification and Control & 14 & & 440 & 869 & 1.98 & 1 \\
\hline 41 & IEEE Trans. on Automation Science and Engineering & 13 & 1.929 & 286 & 808 & 2.83 & 1 \\
\hline 42 & Asian Journal of Control & 13 & 0.562 & 430 & 706 & 1.64 & 1 \\
\hline 43 & Annual Reviews in Control & 12 & 1.109 & 127 & 550 & 4.33 & 1 \\
\hline 44 & Int. Journal of Control Automation and Systems & 11 & 0.590 & 516 & 928 & 1.80 & 1 \\
\hline 45 & Simulation Modelling Practice and Theory & 11 & 0.586 & 562 & 855 & 1.52 & 1 \\
\hline 46 & Measurement \& Control - UK & 11 & 0.493 & 806 & 540 & 0.67 & 1 \\
\hline 47 & Assembly Automation & 11 & 0.382 & 1230 & 876 & 0.71 & 1 \\
\hline 48 & Automation and Remote Control* & 11 & 0.236 & 4003 & 1266 & 0.32 & 1 \\
\hline 49 & Intelligent Automation and Soft Computing & 9 & 0.224 & 360 & 479 & 1.33 & 1 \\
\hline 50 & Journal of Dynamical and Control Systems & 8 & 0.368 & 199 & 366 & 1.84 & 1 \\
\hline
\end{tabular}

Table 1: Some selected journals in the area of automation and control ranked by the h-index as calculated by the ISI Web of Science. IF08 stands for Impact Factor 2008, TA is the total number of articles and TC is the total number of citations for each journal. Both TA and TC were recorded on the date 15.11.2009. DBH signifies the publication level in the Norwegian DBH database. ${ }^{*}$ Publication data for this journal only available from the year 1990. 
Breivik, Hovland and From, "Trends in Research and Publication: Science 2.0 and Open Access"

\begin{tabular}{|c|c|c|c|c|}
\hline$\#$ & Citations & Title & Authors & Journal \\
\hline 1 & 1053 & $\begin{array}{l}\text { Constrained model predictive } \\
\text { control: Stability and optimality }\end{array}$ & Mayne et al. & $\begin{array}{l}\text { AUT, } 2000 \\
\text { Vol. 36, No. } 6\end{array}$ \\
\hline 2 & 822 & $\begin{array}{l}\text { Coordination of groups of mobile autonomous } \\
\text { agents using nearest neighbor rules }\end{array}$ & Jadbabaie et al. & $\begin{array}{l}\text { TAC, } 2003 \\
\text { Vol. } 48, \text { No. } 6\end{array}$ \\
\hline 3 & 590 & $\begin{array}{l}\text { Consensus problems in networks of agents } \\
\text { with switching topology and time-delays }\end{array}$ & Olfati-Saber et al. & $\begin{array}{l}\text { TAC, } 2004 \\
\text { Vol. } 49, \text { No. } 9\end{array}$ \\
\hline 4 & 491 & $\begin{array}{l}\text { Multilevel inverters: A survey of topo- } \\
\text { logies, controls, and applications }\end{array}$ & Rodriquez et al. & $\begin{array}{l}\text { TIE, } 2002 \\
\text { Vol. } 49, \text { No. } 4\end{array}$ \\
\hline 5 & 424 & $\begin{array}{l}\text { The explicit linear quadratic regulator } \\
\text { for constrained systems }\end{array}$ & Bemporad et al. & $\begin{array}{l}\text { AUT, } 2002 \\
\text { Vol. } 38, \text { No. } 1\end{array}$ \\
\hline 6 & 367 & $\begin{array}{l}\text { A new method for the nonlinear } \\
\text { transformation of means and covariances } \\
\text { in filters and estimators }\end{array}$ & Julier et al. & $\begin{array}{l}\text { TAC, } 2000 \\
\text { Vol. } 45, \text { No. } 3\end{array}$ \\
\hline 7 & 364 & $\begin{array}{l}\text { Time-delay systems: An overview of } \\
\text { some recent advances and open problems }\end{array}$ & Richard & $\begin{array}{l}\text { AUT, } 2003 \\
\text { Vol. } 39, \text { No. } 10\end{array}$ \\
\hline 8 & 318 & $\begin{array}{l}\text { A new discrete-time robust stability } \\
\text { condition }\end{array}$ & de Oliveira et al. & $\begin{array}{l}\text { SCL, } 1999 \\
\text { Vol. } 37, \text { No. } 4\end{array}$ \\
\hline 9 & 312 & $\begin{array}{l}\text { Stability of multiagent systems with } \\
\text { time-dependent communication links }\end{array}$ & Moreau et al. & $\begin{array}{l}\text { TAC, } 2005 \\
\text { Vol. 50, No. } 2\end{array}$ \\
\hline 10 & 290 & $\begin{array}{l}\text { Stabilization of linear systems with } \\
\text { limited information }\end{array}$ & Elia et al. & $\begin{array}{l}\text { TAC, } 2001 \\
\text { Vol. } 46, \text { No. } 9\end{array}$ \\
\hline
\end{tabular}

Table 2: Ranking of the highest cited papers (by ISI Web of Science on 17.11.2009) in the top 10 journals from Table 1 during the last decade. AUT=Automatica, TAC=IEEE Transactions on Automatic Control, SCL=Systems \& Control Letters, TIE=IEEE Transactions on Industrial Electronics.

authors obtain more citations and also to obtain them quicker. However, other studies point out alternative explanations to the increase in citations such as selfselection bias since authors typically only distribute their best work, and the early-view effect since online publication dates in general are earlier than for print publications (Frandsen, 2009).

Concerning download statistics, Watson (2009) reports that they provide a useful indicator, two years in advance, of eventual citations, and that correlation between downloads and citations is as high as 0.74 . These results are obtained for the open access Journal of Vision.

In many fields of mathematics and physics almost all scientific papers are placed in the arXiv repository, which is an online archive for electronic preprints. Started back in 1991, the arXiv was one of the precipitating factors that led to the OA movement. Henneken et al. (2006) find that papers submitted to the arXiv are more cited than comparable articles not present in the archive, and also that, in general, the most important papers are submitted to the arXiv before they are published elsewhere.

In the most extensive study to date, covering around 26 million articles from more than 8000 journals which went online before 2006, Evans and Reimer (2009) find that making articles freely available on the web particularly increase the number of citations in developing countries. Given that scientists and researchers from such countries have limited access to the pay-based alternatives, these results are not surprising. Hence, the proliferation of open access publications is expected to contribute strongly to reduce the current information gap which exists between the developed and developing parts of the world.

\subsection{Statistics for 3 Selected OA Journals}

Table 3 shows some statistics for 3 selected OA journals within the fields of modeling, identification and control.

\begin{tabular}{|l|l|l|l|l|l|}
\hline Journal & Est. & TA & TC & AC & h \\
\hline JMLR & 2001 & 626 & 7538 & 837.56 & 37 \\
MIC & 1980 & 440 & 869 & 28.97 & 14 \\
IJCCC & 2006 & 286 & 69 & 17.25 & 3 \\
\hline
\end{tabular}

Table 3: Statistics for 3 different OA journals recorded on 17.11.2009. TA is the total number of articles, TC the total number of citations and AC the average number of citations per year.

The International Journal of Computers, Communications \& Control (IJCCC) is a relatively new OA journal which was started in 2006. With 286 articles and a h-index of only 3, IJCCC illustrates how difficult it is for a newly established OA journal to gain reputation. Having only recently converted to OA, the citation data and h-index for MIC are included for fu- 
ture reference to evaluate the transition effects.

The Journal of Machine Learning Research (JMLR) is an OA journal which was founded in 2000 as an open access alternative to the well-established Machine Learning Journal (MLJ). In 2001, forty editors of MLJ resigned to support JMLR, signing a letter stating that "...our resignation from the editorial board of MLJ reflects our belief that journals should principally serve the needs of the intellectual community, in particular by providing the immediate and universal access to journal articles that modern technology supports, and doing so at a cost that excludes no one. We are excited about JMLR, which provides this access and does so unconditionally."

Hosted at MIT, JMLR is an example of an OA journal in a field close to MIC which has become a high impact publication in a relatively short time. Figures 1 and 2 illustrate its development. While the number of published articles since 2004 has been almost constant at around 80 per year, the number of citations has grown exponentially. JMLR is currently the only level 2 OA journal in the Norwegian DBH database in a field close to MIC. Likely explanations for the rapid and successful development of JMLR are acceptance and support from the research community in machine learning in combination with the quality standards implemented by the editorial team.

\subsection{Print On Demand}

Due to increasing production costs, the financial stability of journals published by scientific societies is in jeopardy and the journals need to find ways to survive (Castillo, 2009): "The problems faced are mainly those of decreasing membership allocations, lack of growth of institutional subscriptions, diminishing advertisements, and pressure for open access. Faced with these situations, a journal can raise subscription rates or switch to an author/institution pay model. With both options, the journal risks alienating its audience and contributors. To reduce cost, some journals opt to become electronic only. Fortunately, a third model exists, and that is print on demand (POD). By using POD, a reader or an institution can pay extra to have a print version of a journal, whereas others may choose online-only subscriptions."

POD is a technology for which new copies of a document are not printed until an order is received. Based on preliminary figures from US publishers, Bowker (2009) projected that US booktitle output in 2008 decreased by $3.2 \%$ to 275232 new titles and editions, down from the 284370 that were published in 2007. Despite the decline in traditional book publishing, there was an extraordinary year of growth in the reported number of POD books published in 2008 .
JMLR: Published Articles Per Year

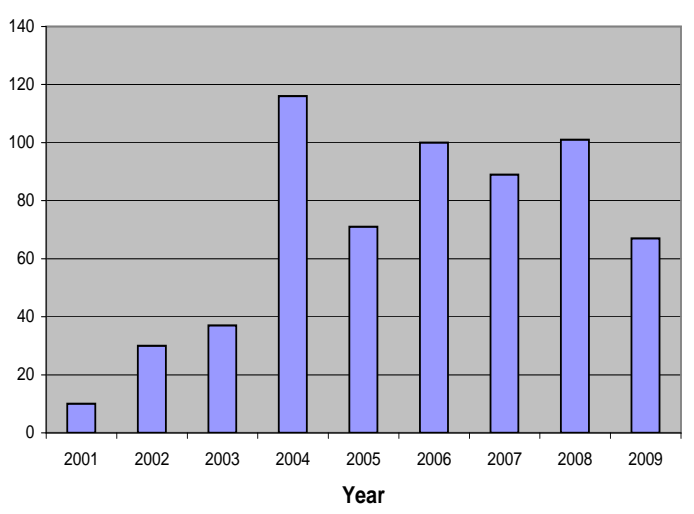

Figure 1: A stabilizing number of published articles for the Journal of Machine Learning Research.

JMLR: Citations Per Year

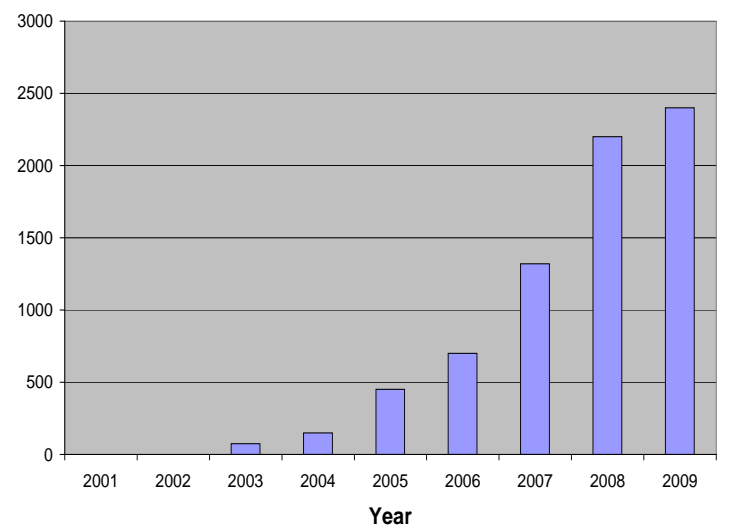

Figure 2: An exponentially increasing number of citations for the Journal of Machine Learning Research.

Bowker projected that 285394 POD books were published this year, a $132 \%$ increase over the 2007 final total of 123276 titles. This was the second consecutive year of triple-digit growth in the POD segment, which in 2008 was $462 \%$ above the 2006 level. The statistics for 2008 benchmark a historic development in the US book publishing industry as the point was crossed where the number of POD books exceeded the number of traditional books entering the market.

POD technology fits particularly well with the online, electronic nature of OA publications by providing a flexible option to produce paper copies on demand, and will probably help further the OA development in the future. As a recent OA journal, MIC was very quick to offer POD as an option for its readers.

\section{New Publication Methods}

Traditionally, the medium of scientific publications has been restricted to paper. Articles have been published in conference proceedings or journals, with strict page 
limitations and formatting rules. Even though electronic technologies render possible a much broader spectrum of publication types, the vast majority of publications are still made as if paper was the only available medium. However, there are signs that this practice is about to change.

SciVee is a video-sharing website for scientists, the science-equivalent of YouTube so to speak, sporting the slogan "Make Your Research Known." Researchers can upload, view and share science-related video clips and connect them to various scientific material through a synchronization feature. For example, synchronization with an article is called a "pubcast" while synchronization with a poster is called a "postercast". Content ranges from the elementary-school level and up to the levels of university and state-of-the-art research.

In (Fink and Bourne, 2007), key contributors from the SciVee team state: "We believe that the research community is ripe for a revolution in scientific communication and that the current generation of scientists will be the one to push it forward. These scientists ... have a natural ability to do science in an electronic environment without the need for printed publications or static documents and, in fact, can feel quite limited by the traditional format of a publication. Perhaps most importantly, they appreciate that the sheer amount of data and the number of publications is prohibitive to the traditional methods of keeping current with the literature." The authors also claim that: "SciVee makes it easier and faster to keep up with current literature by delivering the key points of articles in a portable and enjoyable medium. A reader can interact with several articles using this website in the time it would take to read a single full article in the traditional way." Fink and Bourne then conclude with their belief that: "...revitalizing journal articles will have a significant impact on the scientific community. The traditional article format no longer effectively supports the research in the electronic age."

Conferences are also starting to let authors upload videos and animations in addition to their traditional written contributions. Including MIC, journals now allow color pictures in articles as well as clickable DOI and HTTP links embedded in the PDF files. In the future, even more interactive articles will probably emerge, containing not only text and pictures, but also animations, movies and sound embedded in the documents. As with SciVee, author presentations can then be synchronized with such articles to achieve a maximum dissemination effect. In the electronic world the possibilities thus seem endless. However, care must naturally be taken to develop standards for such content and to uphold scientific quality all along the way.

Interestingly, it seems that scientists from some particular research fields are fronting this development. Founding members of several of the Science 2.0-supporting websites, including OpenWetWare, ResearchGATE, Mendeley and SciVee, have a background from biology and genetics research, where efficient handling of huge amounts of data is required. These scientists have probably felt a special need to change the way research is performed and disseminated in the online world of today.

Although still mostly keeping to traditional publication methods and formats, researchers within the field of automation and control have also begun to take advantage of the possibilities brought on by the web. For example, Karl Åström and Richard Murray have recently authored the book Feedback Systems: An Introduction for Scientists and Engineers and made it freely available through a wiki (Murray, 2009). The wiki also contains lecture materials, source code for relevant software, supplemental examples and exercises, as well as a FAQ database. In addition, the book is available in print for purchase (Aström and Murray, 2008).

In the preface of their book, the authors state that: "This book provides an introduction to the basic principles and tools for the design and analysis of feedback systems. It is intended to serve a diverse audience of scientists and engineers who are interested in understanding and utilizing feedback in physical, biological, information and social systems." Taking note of the fact that research is becoming increasingly multidisciplinary, Åström and Murray have thus written a book which aims to explain the principles of automatic control to a broad audience, and they have chosen to release it through a wiki-based framework which allows for convenient and unrestricted dissemination. Their initiative is in spirit with the concepts of open access and Science 2.0, and hopefully more efforts of a similar kind will be seen in the near future.

\section{Conclusions}

This paper has reviewed some recent trends in research and publication that have the potential to fundamentally transform the way researchers, scientists and engineers carry out their work. New and innovative tools for literature management and research collaboration are currently being introduced, following the development seen in the general population concerning Web 2.0 applications such as blogs, wikis and socialnetworking tools.

While most scientists during the last two decades have been accustomed to perform their work using computers, their methods of publication and collaboration have not adjusted accordingly. However, led by researchers within biology and genetics who daily 
struggle with large amounts of data and a radically increasing body of research literature, new web-based applications are being developed to facilitate efficient online collaboration between scientists around the world. Such a development relies partly on open access publication policies.

Currently, OA publications represent a break with the traditional pay-based channels by making research results freely available on the Internet. These publications increase the online visibility and seem to give a rise in citations compared to their pay-based counterparts. The MIT-hosted journal JMLR demonstrates that new OA journals can rapidly challenge established journals in terms of citations and other ranking indices. Having recently converted to OA, MIC also aims to play a role in this development. Its goal is to constructively contribute within the fields of modeling, identification and control for the benefit of all.

Recognizing the fantastic possibilities brought about by the Internet, the movements of Science 2.0 and Open Access may very well represent the mutually interacting dynamics required to bring science and research into the 21 st century.

\section{References}

Bjørkeng, P. K. Forskere føler at de blir flådd. Aftenposten, 2009. URL http://www.aftenposten.no/ kul_und/article2949887.ece.

Bowker. Statistics on U.S. book publishing for 2008, Press release, May 19. 2009. URL http://www. bowker. com.

Bush, V. As we may think. Atlantic Monthly, July 1945. pages $101-108$.

Castillo, M. Print on demand and scientific publishing. American Journal of Neuroradiology, 2009. 30(1):1. doi:10.3174/ajnr.A1298.

Evans, J. A. and Reimer, J. Open access and global participation in science. Science, 2009. 323(5917):1025. doi:10.1126/science.1154562.

Fink, J. L. and Bourne, P. E. Reinventing scholarly communication for the electronic age. CTWatch Quarterly, 2007. 3(3):26-31.

Frandsen, T. F. Scholarly Communication Changing: The Implications of Open Access. Ph.D. thesis, Royal School of Library and Inf. Science, Denmark, 2009.

Garfield, E. Citation indexes to science: A new dimension in documentation through association of ideas. Science, 1955. 122:108-111. doi:10.1126/science.122.3159.108.
Gripsrud, S. Kjenner ikke BORA. På Høyden, 2006. URL http://nyheter.uib.no/?modus=vis_ nyhet\&id=30523.

Henneken, E. A., Kurtz, M. J., Eichhorn, G., Accomazzi, A., Grant, C., Thompson, D., and Murray, S. S. Effect of e-printing on citation rates in astronomy and physics. J. of Electronic Publ., 2006. 9(2).

Hirsch, J. E. An index to quantify an individual's scientific research output. Proc. National Academy of Sciences of the USA, 2005. 102(46):16569-16572.

Hugubakken, T. Bibliotekene vil ha forskerne med på opprør. Universitetsavisa, 2006. URL http://www . universitetsavisa.no/ua_lesmer. php?kategori=nyheter\&dokid=43c2095edae056. 13683998.

Lawrence, S. Online or invisible? Nature, 2001. 411 (6837): 521.

Lessig, L. TED Talks: Larry Lessig on laws that choke creativity. 2009. URL http://www.ted.com/talks/larry_lessig_ says_the_law_is_strangling_creativity.html.

Murray, R. M. Amwiki. 2009. URL http://www.cds . caltech.edu/ murray/amwiki. [Online; accessed 25-Nov-2009].

Norges Forskningsråd. Forskningsrådets prinsipper for åpen tilgang til vitenskapelig publisering. Vedlegg 3 til KD 29/01/2009, 2009.

Richards, P. Top schools announce joint support for open-access publication. MIT media relations, 2009. URL http://web.mit.edu/press/ 2009/open-access-091409.html.

Waldrop, M. M. Science 2.0: Great new tool, or great risk? Scientific American, 2008. URL http:// www.scientificamerican.com/article.cfm?id= science-2-point-0-great-new-tool-or-great-risk.

Watson, A. B. Comparing citations and downloads for individual articles. Journal of Vision, 2009. 9(4):14. doi:doi:10.1167/9.4.i.

Wikipedia. Web 2.0 - Wikipedia, The Free Encyclopedia. 2009a. URL http://en.wikipedia.org/ w/index.php?title=Web_2.0\&oldid=326313261.

[Online; accessed 17-Nov-2009].

Wikipedia. YouTube - Wikipedia, The Free Encyclopedia. 2009b. URL http://en.wikipedia.org/ w/index . php?title=YouTube\&oldid=325665878.

[Online; accessed 17-Nov-2009].

Åström, K. J. and Murray, R. M. Feedback Systems: An Introduction for Scientists and Engineers. Princeton University Press, 2008. 